\title{
CONTROLADOR ROBUSTO QFT DE TEMPERATURA EN TIEMPO REAL
}

\section{ROBUST CONTROL QFT FOR TEMPERATURE IN REAL TIME}

\author{
MSc(c). Rafael Augusto Núñez Rodríguez*, Ing. Lisseth Bohórquez Ramírez*, \\ Ing. Robert Martinez Mora*
}

* Unidades Tecnológicas de Santander, Facultad de Ciencias Naturales e Ingenierías, Grupo de Investigación en Control Avanzado - GICAV.

Calle de los Estudiantes \# 9-82 Real de Minas, Bucaramanga, Santander, Colombia. Tel.: 57-7-6917700, Fax: 57-7- 6917691, Ext. 2007

E-mail: \{rrodriguez, lbohorquez, rmartinez\}@ correo.uts.edu.co

\begin{abstract}
Resumen: Se presenta el diseño de un controlador robusto basado en la técnica QFT para el control de la temperatura interna de un horno eléctrico, que permita estabilidad robusta con base en el margen de ganancia y margen de fase, y rechazo de perturbaciones a la salida del sistema. Se implementó un sistema de adquisición de datos en tiempo real para capturar la variable de temperatura y variar la potencia promedio aplicada a la resistencia de calefacción, en función de la señal de control. El controlador basado en la teoría de realimentación cuantitativa se validó respecto un controlador PID a partir de índices de desempeño y respuesta transitoria, ante cambios en el punto de operación y perturbaciones de carga en la salida de la planta de temperatura.
\end{abstract}

Palabras clave: Control QFT, Control robusto, Control de temperatura, Control PID.

\begin{abstract}
This work shows the design of a robust controller based on QFT technique to control the internal temperature of an electric oven. The design allows robust stability, based on the gain margin and phase margin, and disturbance rejection at the output of the system. It was implemented the data acquisition system in real time to measure the temperature variable and to modify the average power applied to the heating resistor, given the signal control. The QFT controller was compared with a PID controller from performance indices and transient response, to changes in the operation point and, disturbances in the output payload of the electric oven system.
\end{abstract}

Keywords: QFT control, Robust control, Temperature control, PID control.

\section{INTRODUCCIÓN}

La temperatura es uno de las variables de proceso ampliamente estudiada y controlada a nivel industrial dada su influencia sobre el desempeño del proceso (Sánchez, 2006), incluso se considera un factor importante en aplicaciones para la producción de biogás a partir de digestión anaeróbica(Criollo, Alvarado, \& Numpaque, 2014). Se han desarrollado diferentes técnicas de control para mitigar los efectos del tiempo muerto, típicos en la dinámica de este tipo de proceso, tal como un controlador predictivo basado en modelo, el cual presentó un excelente desempeño respecto a la respuesta de un controlador de temperatura PID para un calentador eléctrico (Hernández Arroyo, Díaz Rodríguez, \& Pinzón Ardila, 2011). Por otra parte, el problema de control se han enfocado a establecer algoritmos de control para mejorar la respuesta dinámica del proceso, tal como un esquema de control de matriz dinámica acoplado a un controlador PID, que permitió mayor frecuencia de muestreo con el fin de mitigar los efectos de perturbaciones externas (Luo \& Wu, 2004). 
Técnicas de control basadas en lógica Fuzzy acoplado a un modelo de predictor de Smith, permiten optimizar la utilización de combustible en hornos que gran capacidad cuyo tiempo muerto es un parámetro que afecta el desempeño del controlador (Gonzalez, Perez, Batlle, Fernandez, \& Perez, 2016).

En este artículo se presenta el diseño de un controlador robusto basado en la técnica QFT para el control de la temperatura interna de un horno eléctrico, que permita estabilidad robusta con base en el margen de ganancia y margen de fase, y rechazo de perturbaciones a la salida del sistema. Mplo. La Teoría de Realimentación Cuantitativa, conocida más comúnmente como QFT (Quantitative Feedback Theory) (Houpis, Rasmussen, \& Garcia-Sanz, 2005), e inicialmente desarrollada por I. Horowitz, presenta una gran versatilidad a la hora de sintonizar controladores robustos para el control de temperatura de process, dada la capacidad de gobernar el proceso a pesar de las incertidumbres existentes.

\section{MATERIALES Y MÉTODOS}

\subsection{Modelamiento}

Para intercambiar datos con el sistema de calentamiento y establecer el controlador en tiempo real, se implementó un sistema de adquisición de datos a partir de la tarjeta PCI-6014 conectada a un computador compatible con LabView $\AA$, que permite monitorear y controlar las variables del proceso en una misma plataforma virtual. El transmisor de temperatura PT-100 con salida 4$20 \mathrm{~mA}$ ubicado al interior del horno, se conecta a la entrada analógica ACH4 de la tarjeta PCI-6014 por medio del convertidor corriente a voltaje XTR111 de Texas Instruments ${ }^{\circledR}$. El relé de estado sólido permite variar el voltaje RMS aplicado a las resistencias de calefacción, y de esta forma variar la cantidad de calor generado al interior del horno (García, García, \& Amorós, 1999). Éste es conectado a la tarjeta de adquisición de datos en la salida analógica DAC0OUT, en la cual se genera la señal PWM en función de la señal de control. En la figura 1 se ilustra el diagrama de bloques del proceso.

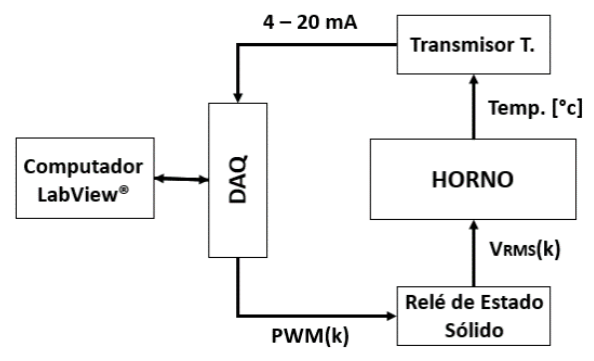

Fig. 1. Esquema de controlador en tiempo real

El modelamiento matemático de la planta se realizó a partir de la curva de reacción de la temperatura, en lazo abierto, ante una entrada tipo escalón del $50 \%$ de ciclo útil de la señal PWM aplicada al relé de estado sólido. La respuesta de la planta se aproxima a un sistema de primer orden con retardo, cuyos parámetros se determinan utilizando la técnica propuesta por Cecil L. Smith (Sánchez, 2006). La constante de tiempo $T$ del sistema se determina a partir la ecuación (1), que depende de los tiempos en los cuales el sistema alcanza el $28.3 \%$ y el $63.3 \%$ del valor final de temperatura.

$T=\frac{3\left(t_{63.2 \%}-t_{28.3 \%}\right)}{2}$

El tiempo muerto se calcula a partir del tiempo en el cual el sistema alcanza el $63.2 \%$ del valor final, y de la constante de tiempo del sistema, tal como se observa en la ecuación (2). La ganancia estática está relacionada con la variación de la señal de salida en estado estable respecto a una variación en la señal de entrada de la planta. Ésta se determina de acuerdo a la ecuación (3).

$$
\begin{array}{r}
L=t_{63.2 \%}-T \\
K=\frac{\Delta Y}{\Delta P W M}=\frac{T_{\max }-T_{\min }}{P W M_{\max }-P W M_{\min }}
\end{array}
$$

El sistema de primer orden con retardo se define de acuerdo a la ecuación (4), donde el tiempo muerto $L=120.5 \mathrm{~s}$, la constante de tiempo del sistema $T=342.9 \mathrm{~s}$, y la ganancia estática $K=4.027$.

$G p(s)=\frac{K e^{-L s}}{T s+1}$ 


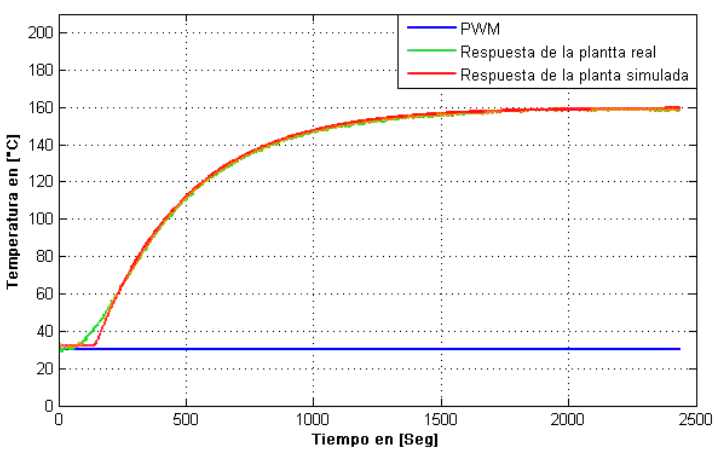

Fig. 2. Respuesta del modelo matemático de la planta.

Se validó el modelo identificado respecto a la respuesta real del sistema, tal como se muestra en la figura 2. Se pudo demostrar que el modelo identificado se ajusta a la respuesta del sistema real, para este punto de operación.

\subsection{Diseño controlador QFT de temperatura}

\subsubsection{Espacio de Incertidumbre}

Definir el espacio de incertidumbre es uno de los aspectos más relevantes para plantear el diseño de un controlador QFT (Houpis et al., 2005). Para determinar la variación paramétrica del proceso, se realizaron 10 experimentos de excitación e identificación de la planta con el fin de encontrar el intervalo de variación de los parámetros de la función de transferencia de primer orden con retardo dada por la ecuación (4). Se pudo establecer una variación considerable respecto a los parámetros identificados ante una entrada escalón del 50\% de ciclo útil de la señal PWM. Estos datos se muestran en la Tabla 1.

Tabla 1: Variación de Parámetros

\begin{tabular}{cccc}
\hline Parámetro & $\boldsymbol{m i n}$ & $\boldsymbol{m a x}$ & Nominal \\
\hline$K$ & 2.875 & 4.8 & 3.6 \\
$T$ & $213.9 \mathrm{~s}$ & $406.4 \mathrm{~s}$ & $305.2 \mathrm{~s}$ \\
$L$ & $97.1 \mathrm{~s}$ & $145.8 \mathrm{~s}$ & $120.1 \mathrm{~s}$ \\
\hline
\end{tabular}

Con base en los datos de la Tabla 1, se establece un intervalo de incertidumbre para la ganancia $K$, $\Delta K=\left[\begin{array}{ll}2.875 & 4.8\end{array}\right] \forall K \in \mathbb{R}$, para la constante de tiempo $T, \Delta T=[213.9 s 406.4 s] \forall T p \in \mathbb{R}$, y el tiempo muerto $\Delta T=\left[\begin{array}{l}97.1 s \\ 145.8 s\end{array}\right] \forall T p \in \mathbb{R}$, el cual permitió generar una familia de plantas evaluada ante un conjunto de frecuencias de interés entre $0.0001 \mathrm{rad} / \mathrm{s}$ a $0.1 \mathrm{rad} / \mathrm{s}$, dado el ancho de banda del sistema, obteniendo para cada frecuencia una representación fase $\left[{ }^{\circ}\right]$ - magnitud $[\mathrm{dB}]$ del conjunto de plantas sobre el diagrama de Nichols
(Houpis et al., 2005), tal como se muestra en la Fig. 3.

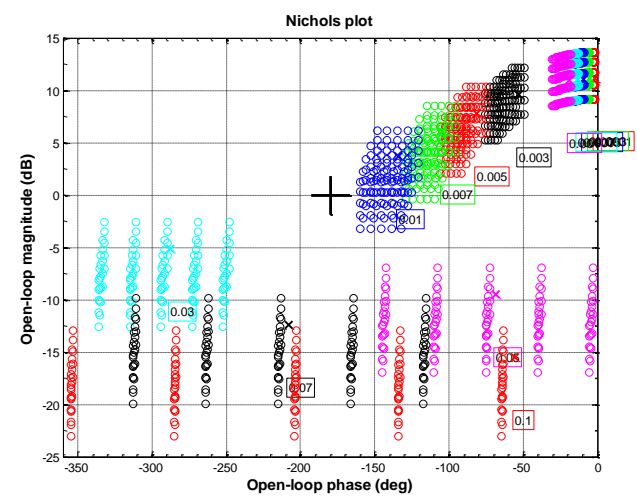

Fig. 3. Espacio de Incertidumbre paramétrica

\subsubsection{Especificaciones de desempeño del controlador}

Dado que el sistema de temperatura es sometido a perturbaciones externas a la salida del proceso, y presenta variación en los parámetros a causa de las diferentes condiciones ambientales, se definieron dos especificaciones de desempeño basados en la estabilidad robusta mínima recomendada de $5 \mathrm{~dB}$ en ganancia y $45^{\circ}$ de fase (Martínez, 2001), y rechazo de perturbaciones de carga en la temperatura al interior del horno, las cuales según la teoría de realimentación cuantitativa se definen en la ecuación (5) y la ecuación (6).

$$
\begin{aligned}
& \left|\frac{\mathrm{y}}{r}\right|=\left|\frac{L(j \omega)}{1+L(j \omega)}\right|<\delta_{u}(\omega) \\
& \left|\frac{y}{d}\right|=\left|\frac{1}{1+L(j \omega)}\right|<\delta_{S}(\omega)
\end{aligned}
$$

Las inecuaciones dadas en la ecuación (5) y la ecuación (6), se resuelven a partir de los valores de los parámetros $\delta_{u}(\omega)$, y $\delta_{s}(\omega)$, bien sea como constantes o a partir de funciones de transferencia que representen la dinámica deseada de la planta. El criterio de estabilidad robusta se define a la partir de la ecuación $(5)$, con $\delta_{u}(\omega)=1.3$, dando como resultado un margen de fase de $45^{\circ}$ y margen de ganancia $5 \mathrm{~dB}$, de acuerdo a la ecuación (7) y la ecuación (8).

$$
\begin{gathered}
M F \geq 180^{\circ}-\arccos \left(\frac{0.5}{\delta_{U}}-1\right) \\
M G \geq 1+\frac{1}{\delta_{U}}
\end{gathered}
$$


El rechazo de perturbaciones a la salida de la planta de temperatura, se define a partir del parámetro $\delta_{S}(\omega)$ y la ecuación (6) (Elso, Gil-Martinez, \& Garcia-Sanz, 2017). Éste se define como una función de transferencia que representa la dinámica de la planta ante una perturbación de temperatura. Esta respuesta está sujeta a la dinámica del sistema real, por tanto se definió un tiempo de establecimiento de la salida ante una perturbación tipo escalón unitario, de $1500 \mathrm{~s}$.

Para definir la función de transferencia $\delta_{s}(\omega)$, se aplicó el método de asignación de polos (Houpis, Sheldon, \& D’Azzo, 2003), cuya resultante se muestra en (9).

$$
\delta_{S}(\omega)=\frac{s^{2}+0.001997 s}{s^{2}+0.003994 s+3.989 \times 10^{-6}}
$$

\subsubsection{Conformación de Contornos}

Con base en las especificaciones de desempeño del controlador dadas en la ecuación (5) y la ecuación (6), y de la función de transferencia que representa el parámetro $\delta_{s}(\omega)$ dado en la ecuación (9), se debe conseguir una $L(j \omega)$ tal que cumpla las desigualdades establecidas en la ecuación (5) y ecuación (6), donde $L(j \omega)=G(j \omega) P(j \omega)$. Así, el problema de control se centra en determinar un único controlador $G(j \omega)$ que cumpla con todas las especificaciones de desempeño establecidas a partir de la planta con incertidumbre $P(j \omega)$ en el rango de frecuencias de interés (Gil-Martínez \& GarcíaSanz, 2003).

Para resolver el problema de control, se plantea una inecuación cuadrática por cada especificación de desempeño (Chait \& Yaniv, 1993), tal como se muestra en la ecuación (10) y la ecuación (11).

$$
\begin{aligned}
& p^{2}\left(1-\frac{1}{\delta_{u}^{2}}\right) g^{2}+2 p \cos (\emptyset+\theta) \mathrm{g} \geq 0 \\
& p^{2} g^{2}+2 p \cos (\emptyset+\theta) \mathrm{g}+\left(1-\frac{1}{\delta_{s}^{2}}\right) \geq 0
\end{aligned}
$$

Las inecuaciones cuadráticas (10) y (11) se resuelve a partir de métodos iterativos de acuerdo al algoritmo propuesto por (Chait \& Yaniv, 1993), donde $g$ equivale a la representación polar del controlador y $p$ representa la forma polar de la planta nominal del lazo $L_{0}$.

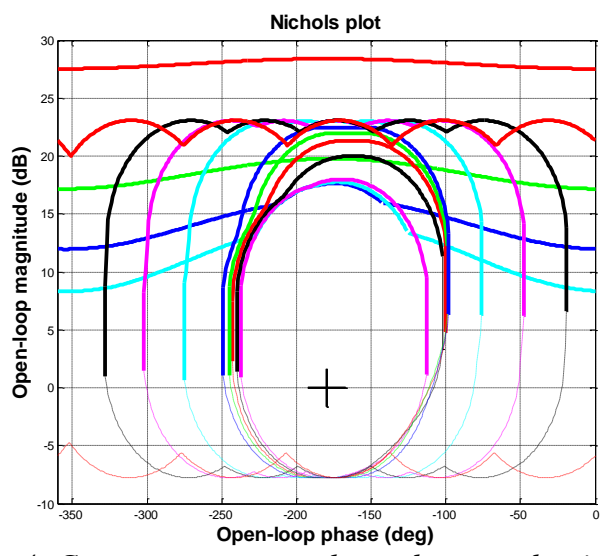

Fig. 4. Contornos para rechazo de perturbaciones a la salida y estabilidad robusta

Cada raíz del sistema de inecuaciones cuadráticas representa un lugar en el diagrama de Nichols para cada par fase $\left[^{\circ}\right]$ - magnitud $[d B]$, para las $n$ frecuencias de interés y la planta nominal, donde se generan $n$ cantidad de puntos los cuales representan los contornos de cada especificación, de manera que el único controlador $g e^{j \emptyset}$ que cumple con todas las especificaciones de desempeño es aquel que consiga llevar la función del lazo $L$ encima de los intersecciones de todos los contornos de cada especificación (Houpis et al., 2005). Los intercepto de los contornos para las especificaciones de desempeño de controlador propuesto, se muestran en la Fig. 4.

\subsubsection{Loop-shapping del controlador QFT}

Mediante la técnica loop-shapping, se introduce un controlador $G(s)$ que manipule la función de lazo $L_{o}$ hasta lograr cumplir con las restricciones impuestas por las especificaciones de desempeño, trazada en los contornos de la figura 4. La respuesta de $L_{o}$ en la frecuencia de interés debe quedar por encima del intercepto de los contornos en cada frecuencia de interés, tal como se muestre en la figura 5. Esto se logra agregando polos y ceros a la función de lazo $L_{o}$ hasta conseguir la respuesta deseada (Martínez, 2001). La función de transferencia del controlador diseñado se muestra en la ecuación (12).

$\mathrm{G}(s)=\frac{1.822 s^{2}+0.2596 s+0.00084}{s\left(0.001994 s^{2}+0.08931 s+1\right)}$

Para construir el controlador de la ecuación (12), se agregó un integrador, un polo complejo con frecuencia natural de $22.4 \mathrm{rad} / \mathrm{s}$ y amortiguamiento relativo de 1 , y se agregó además, dos ceros reales, a la función del lazo nominal $L_{o}$. 


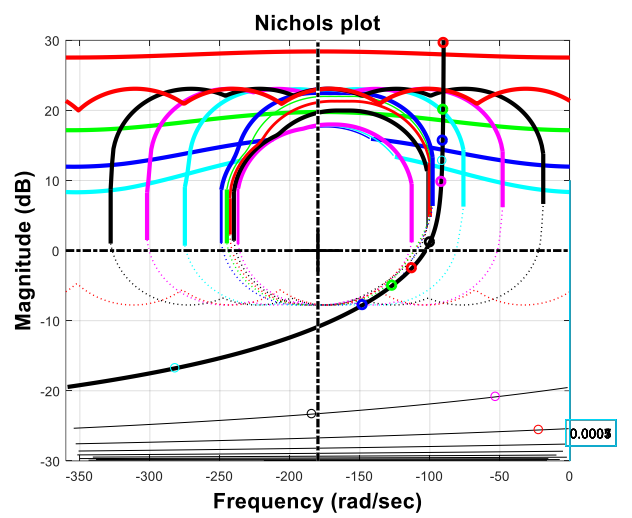

Fig. 5. Respuesta del Controlador QFT con $L_{o}$.

Dado que se propuso un esquema capaz de rechazar perturbaciones de carga, es necesario analizar la respuesta del controlador ante perturbaciones a la salida del proceso. La dinámica del proceso ante perturbaciones externas se modela de acuerdo a la ecuación (9) y la especificación está dada por la ecuación (11), así, se debe buscar que la respuesta en frecuencia de la función $L$ se encuentre condicionada a la dinámica del modelo de la ecuación (9). El controlador de la ecuación (12), permitió que la respuesta en el dominio del tiempo se ajuste a los límites impuestos por la función $\delta_{s}(\omega)$. La respuesta de la familia de funciones $L$, ante una perturbación de carga a la salida tipo escalón unitario, se presenta en la figura 6 , donde la curva roja, representa la respuesta de la planta nominal $L_{o}$.

\section{ANÁLISIS DE RESULTADOS}

Se diseñó e implementó un sistema experimental para la adquisición de datos en tiempo real de la planta sujeta a estudio. La acción de control se codificó en una señal por modulación de ancho de pulso (PWM), para determinar los tiempos de encendido y apagado del relé de estado sólido, controlando de esta forma la variable de la temperatura en el horno eléctrico. Utilizando el software LabView ${ }^{\circledR}$ se pudo implementar el algoritmo de control en tiempo real para interactuar físicamente con la variable temperatura.

En la figura 6 se muestra la respuesta del sistema para una referencia de $150^{\circ} \mathrm{C}$, un sobreimpulso de $6^{\circ} \mathrm{C}$, con el cual alcanza una temperatura máxima de $156^{\circ} \mathrm{C}$, y un tiempo de establecimiento de aproximadamente $1800 \mathrm{~s}$.

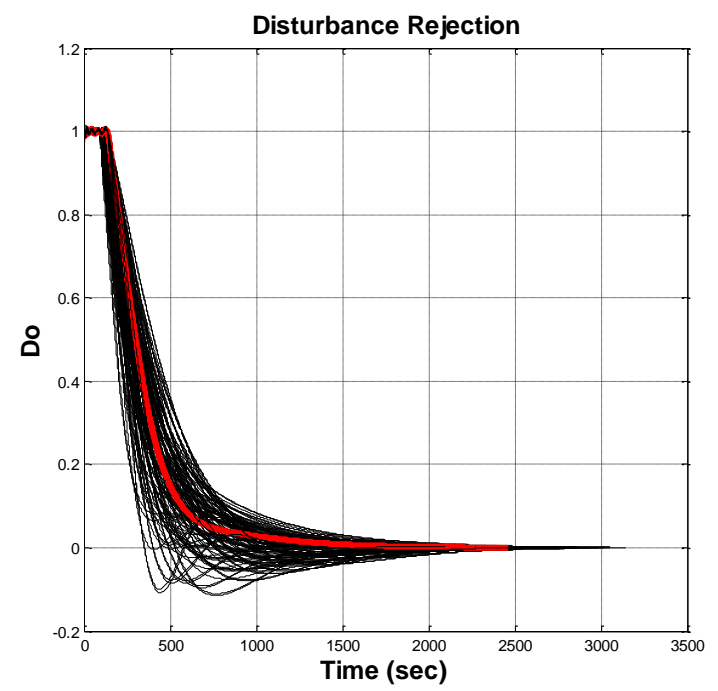

Fig. 6. Respuesta del Controlador QFT ante perturbaciones de carga.

La figura 7 muestra la respuesta real y la respuesta simulada del sistema con el esquema de control QFT. Se observa que la respuesta dinámica del sistema simulado se aproxima a la respuesta del sistema real, lo que permite validar el modelo de simulación y la estrategia de control. La respuesta real tuvo un sobrepaso del $4 \%$, igual a la simulada, pero el sistema real fue un poco más lento respecto la respuesta simulada. El tiempo de asentamiento para ambas respuestas fue aproximadamente de $1800 s$, el comportamiento de las señales de control fue similar, con una pequeña diferencia de $2 \%$ estabilizándose en $\pm 30 \%$ de ciclo útil.

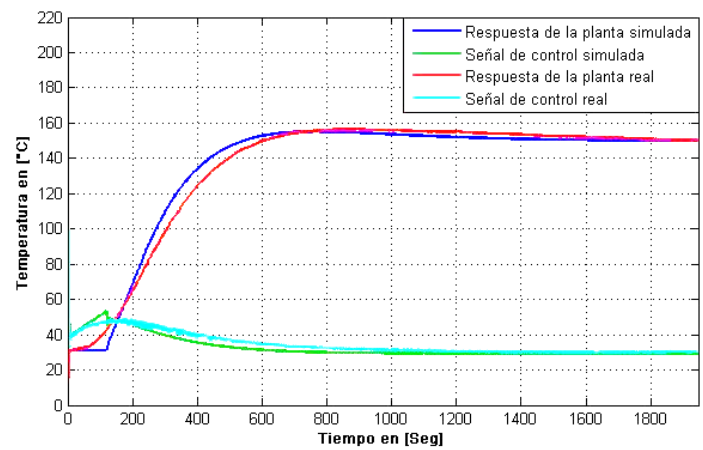

Fig. 7. Respuesta del sistema real y sistema simulado en lazo cerrado.

Se pudo establecer una comparación comparación de un controlador clásico PID sin acción antiwidup, dadas las limitaciones del actuador y los efectos del integrador, y el controlador QFT, para una referencia de $180^{\circ} \mathrm{C}$. En la figura 8 se muestra esta comparación. 


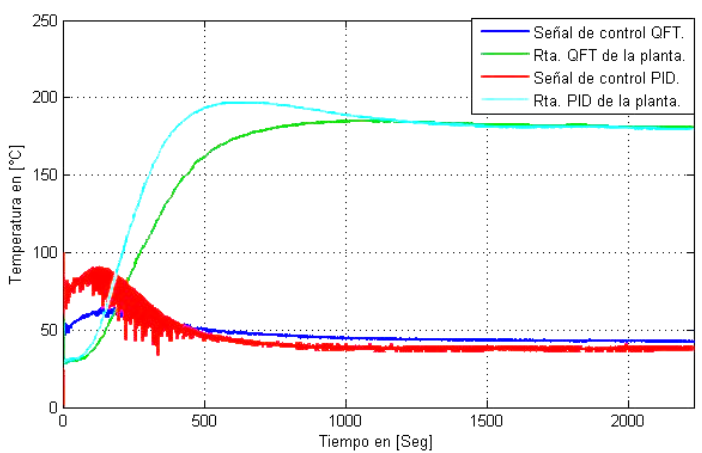

Fig. 8. Comparación respuesta de la planta con controlador PID y controlador QFT.

En la figura 8 se aprecia la respuesta del sistema con ambos controladores, donde el PID obtuvo un sobrepaso del $10 \%$ mientras que el controlador QFT fue de 5\%, además tuvo un mayor esfuerzo en la señal de control del controlador PID a causa de los cambios bruscos aplicados al actuador y mayor área bajo la curva roja de la figura 8 por su alta sensibilidad al ruido en el sensor, mientras que el controlador QFT fue más eficiente, de que ambos controladores presentaron un tiempo de establecimiento de $1200 \mathrm{~s}$ con el mismo porcentaje de ciclo útil aplicado al actuador.

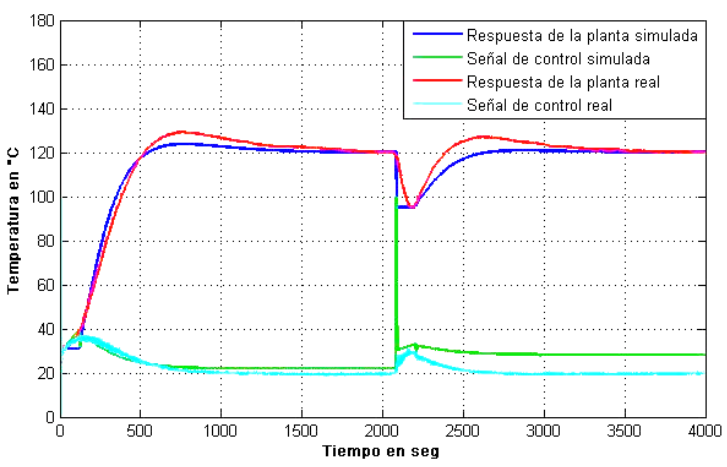

Fig. 9. Respuesta del sistema ante una perturbación real y simulada.

Se validó la respuesta del controlador QFT ante una variación externa de la temperatura al interior del horno. Esta perturbación se introdujo a sistema al abrir la tapa del horno a los 2085s de haber comenzado la prueba, y ésta se comparó con su respuesta simulada. En la figura 9 se observa la gráfica entre la respuesta simulada y la real, donde se observa que la respuesta real alcanzó un sobrepaso al iniciar la prueba y al haber terminado con la perturbación, además su señal de control se asemeja a su contraparte simulada.

Cabe destacar que el modelo matemático no tiene en cuenta la dinámica de enfriamiento del horno y por esto se observa que la respuesta simulada tiene un cambio abrupto de temperatura que su contraparte real, pero en términos generales la respuesta se asemeja a la respuesta simulada.

\section{CONCLUSIONES}

El controlador QFT propuesto para el rango de incertidumbre de los parámetros del sistema de temperatura, en presencia de tiempo muerto, mitigó los efectos de las perturbaciones externas en el menor tiempo posible con el mínimo esfuerzo de la señal de control.

Diseñar un controlador robusto QFT en el dominio de la frecuencia es una metodología donde se deben dominar funciones de sensibilidad y sensibilidad complementaria relacionadas con el lazo de control realimentado, las cuales permiten que el sistema de temperatura pueda ejercer rechazo de perturbaciones, ser robusto ante el ruido del sensor y reducir energía de control a pesar de la variación de fase a causa del tiempo muerto.

La realimentación redujo el efecto de las perturbaciones en el comportamiento del sistema, permitiendo que la variable del proceso fuera igual a la señal de referencia a pesar de las variaciones constantes que representan dichas perturbaciones.

\section{REFERENCIAS}

Chait, Y., \& Yaniv, O. (1993). Multi-input/singleoutput computer-aided control design using the quantitative feedback theory. International Journal of Robust and Nonlinear Control, 3(1), 47-54. https://doi.org/10.1002/rnc.4590030103

Criollo, B., Alvarado, J. D., \& Numpaque, H. (2014). Control PID de temperatura y dosificación de ph para la producción de gas metano a partir de la digestión anaeróbica de residuos sólidos orgánicos. Revista Colombiana de Tecnologías de Avanzada, Volumen 2(24), 134-141.

Elso, J., Gil-Martinez, M., \& Garcia-Sanz, M. (2017). Quantitative feedback control for multivariable model matching and disturbance rejection. International Journal of Robust and Nonlinear Control, 27(1), 121-134. https://doi.org/10.1002/rnc.3563

Gil-Martínez, M., \& García-Sanz, M. (2003). Simultaneous meeting of robust control specifications in QFT. International Journal of Robust and Nonlinear Control, 13(7), 643-656. 
Gonzalez, I. O. B., Perez, R. R., Batlle, V. F., Fernandez, L. P. S., \& Perez, L. A. S. (2016). Fuzzy Gain Scheduled Smith Predictor for Temperature Control in an Industrial Steel Slab Reheating Furnace. IEEE Latin America Transactions, 14(11), 4439-4447. https://doi.org/10.1109/TLA.2016.7795812

Hernández Arroyo, E., Díaz Rodríguez, J., \& Pinzón Ardila, O. (2011). Comparison of different control techniques applied to real time temperature control. Revista Colombiana de Tecnologías de Avanzada, Volumen 2(18), 94101.

Houpis, C. H., Rasmussen, S. J., \& Garcia-Sanz, M. (2005). Quantitative Feedback Theory: Fundamentals and Applications, Second Edition. CRC Press.

Houpis, C. H., Sheldon, S. N., \& D’Azzo, J. J. (2003). Linear Control System Analysis and Design: Fifth Edition, Revised and Expanded. CRC Press.
Luo, G., \& Wu, G. (2004). Application of transparent control on temperature control system. En Fifth World Congress on Intelligent Control and Automation (IEEE Cat. No.04EX788) (Vol. 1, p. 620-623 Vol.1). https://doi.org/10.1109/WCICA.2004.1340652

Martínez, M. G. (2001). Síntesis de controladores robustos mediante el análisis de la compatibilidad de especificaciones $e$ incertidumbre

(http://purl.org/dc/dcmitype/Text). Universidad Pública de Navarra. Recuperado a partir de https://dialnet.unirioja.es/servlet/tesis?codigo $=1$ 7821

Sánchez, J. A. (2006). Instrumentación y control avanzado de procesos. Ediciones Díaz de Santos. 